\title{
Analysis of nonlinear transport in Couette flow
}

\author{
Chang Sub Kim and James W. Dufty \\ Department of Physics, University of Florida, Gainesville, Florida 32611 \\ Andrés Santos and J. Javier Brey \\ Departamento de Física Teórica, Universidad de Sevilla, Apartado de Correos 1065, Sector Sur, \\ E-41080 Sevilla, Spain \\ (Received 26 June 1989)
}

\begin{abstract}
The nonlinear Bhatnagar, Gross, and Krook [Phys. Rev. 94, 511 (1954)] kinetic equation is solved for boundary conditions leading to planar Couette flow and heat transport. In the limit of zero Knudsen number but arbitrary uniformity parameter (shear rate), an exact "normal" solution is obtained. The velocity distribution function is illustrated explicitly for states far from equilibrium. At finite Knudsen number, the distribution function is obtained from numerical solution of a set of nonlinear, singular integral equations. Results are presented for a range of values of the Knudsen number and uniformity parameter. The approach to a normal state is studied with a determination of the hydrodynamic profiles, velocity slip, shear viscosity, and a nonlinear Burnett transport coefficient.
\end{abstract}

\section{INTRODUCTION}

The study of transport processes in fluids far from equilibrium is difficult for several reasons. Typically, the nonequilibrium state results from boundary conditions that introduce boundary layers inside of which the system cannot be described by hydrodynamics. ${ }^{1}$ Away from the boundary layers hydrodynamics may be applicable, but generally the consitutive equations are highly nonlinear and not well known for conditions far from equilibrium. The boundary-layer problem can be minimized by considering dense fluids, as in some nonequilibrium computer simulations. ${ }^{2}$ However, the theory of nonequilibrium transport for dense fluids is not well developed, and the analysis of such simulations is difficult. More recently, both molecular-dynamics ${ }^{3}$ and Monte Carlo simulations ${ }^{4}$ have been performed for nonequilibrium states at low densities where the theory is better understood. In a series of recent papers ${ }^{5-8}$ we have studied this problem using the model nonlinear Bhatnagar, Gross, and Krook (BGK) equation ${ }^{9}$ that represents many of the qualitative and quantitative features of the nonlinear Boltzmann equation. In particular, an exact solution at zero Knudsen number ${ }^{7}$ and a numerical solution at finite Knudsen number ${ }^{8}$ were given for heat transport. The objective here is to report similar results for the case of planar Couette flow. Such exact results, even for idealized models, provide important examples for controlled comparison with computer simulations and more general theoretical methods.

A primary motivation for this work is to understand better the conditions under which a "normal" state exists. A normal state is a nonequilibrium state for which the space and time dependence of relevant properties (the velocity distribution and its moments) occur entirely through the hydrodynamic variables (e.g., temperature, pressure, and flow velocity). ${ }^{10,11}$ The boundary layers are clearly domains for which the concept of a normal solution does not apply. Qualitatively, boundary layers occur within a few mean free paths of the physical boundaries, as measured by the Knudsen number $\lambda$ (ratio of the local mean free path to the system size). The normal state is characterized by a uniformity parameter which measures the mean free path relative to the spatial variation of the hydrodynamic variables. A normal-state description is expected to apply (if at all) only far from the walls and under conditions of small $\lambda$.

In practical circumstances, the spatial gradients are established by fixing different values for the hydrodynamic variables at the boundaries. Increasing the size of the system to obtain small $\lambda$ for fixed boundary conditions also has the effect of decreasing the uniformity parameter, yielding a normal state that is asymptotically close to equilibrium. It is possible that normal states exist only in this limit, and that states far from equilibrium would necessarily entail a dependence on both uniformity parameter and $\lambda$ even in the bulk. For the special case of heat flow, ${ }^{7,8}$ this question was addressed by increasing the temperature difference at the walls as the system size was increased. In this way the limit of small $\lambda$ could be studied for fixed uniformity parameter. It was found that a normal state applies even for states far from equilibrium under the appropriate conditions. The same conclusions are found for the Couette flow considered here.

In the next section a formal solution to the nonlinear BGK equation is obtained for boundary conditions leading to combined planar Couette flow and heat transport. The boundary conditions are such that the Knudsen number vanishes near the walls, eliminating the boundary layer. An exact normal solution is then obtained by ex- 
ploiting the symmetry of the problem to "guess" the appropriate hydrodynamic fields. The results here for the hydrodynamic fields agree with and justify those of a previous formal analysis. ${ }^{6}$ The velocity distribution function is also constructed explicitly and evaluated under conditions including large shear rate. Thus we obtain an example of a normal solution applicable even to states far from equilibrium. In Sec. III, the more general case of finite Knudsen number is considered and the problem is reduced to solution of a coupled set of five singular, nonlinear, integral equations. A numerical solution to these equations is obtained over a range of Knudsen numbers and uniformity parameters. This range complements the results of Sec. II, since the numerical method becomes inefficient at very small Knudsen numbers. The hydrodynamic fields, velocity slip coefficient, shear viscosity, and velocity distribution function are all in good agreement with the Chapman-Enskog normal solution for points far from the boundaries and for small uniformity parameters. A nonlinear (Burnett order) transport coefficient associated with the stress tensor is calculated as a function of the Knudsen number, with results indicating a normal state beyond Navier-Stokes order. Previous studies of this problem using the BGK equation focused primarily on larger Knudsen numbers ${ }^{12}$ or states near equilibrium. ${ }^{13}$ The conclusions drawn from these analytical and numerical studies are discussed in the last section.

\section{AN EXACT NORMAL SOLUTION}

In this section a formal solution to the stationary state BGK equation is obtained, incorporating the specified boundary conditions. For planar geometry, and idealized boundary conditions (zero-temperature walls), an exact solution is obtained corresponding to Couette flow with associated heat transport. In this special case the boundary layer vanishes, since the Knudsen number vanishes at the walls, so the result is a normal solution.

The BGK kinetic equation and its relationship to the Boltzmann equation has been discussed elsewhere. ${ }^{11}$ It determines the distribution of velocities, $f(\mathbf{v}, \mathbf{r}, t)$, in a gas as a function of space and time for given initial and boundary conditions. Here, we restrict attention to boundary-driven stationary states with spatial variation in only one direction (chosen to be the $x$ direction). Then the BGK equation has the simple form

$$
v_{x} \frac{\partial f}{\partial x}=-v(x)\left(f-f_{L}\right)
$$

The collision frequency $v(x)=v(T(x), p(x))$ depends on $x$ only through its dependence on temperature $T(x)$ and pressure $p(x)$. The latter dependence is determined by the particular interparticle force law considered. The local equilibrium distribution function $f_{L}$ is a nonlinear functional of $f$ through its dependence on $T(x), p(x)$, and the flow velocity $\mathbf{U}(x)$ :

$$
f_{L}(\mathbf{v}, x)=\Theta\left(L^{2}-x^{2}\right) \frac{p(x)}{T^{5 / 2}(x)(2 \pi)^{3 / 2}} \exp \left\{-[\mathbf{v}-\mathbf{U}(x)]^{2} / 2 T(x)\right\} .
$$

We use units such that Boltzmann's constant and the mass equal one. The factor of $\Theta\left(L^{2}-x^{2}\right)$ in Eq. (2.2), where $\Theta$ is the Heaviside step function, reflects the fact that the system is defined for $-L<x<L$. It plays no role in this domain but is convenient for the representation of the solution given below. The hydrodynamic variables, $T(x), p(x)$, and $\mathbf{U}(x)$ are functionals of $f$ through the definitions

$$
\begin{aligned}
& p(x) / T(x)=\int d \mathbf{v} f(\mathbf{v}, x), \\
& \frac{3}{2} p(x)=\int d \mathbf{v} \frac{1}{2}(\mathbf{v}-\mathbf{U})^{2} f(\mathbf{v}, x), \\
& p(x) \mathbf{U}(x) / T(x)=\int d \mathbf{v} \mathbf{v} f(\mathbf{v}, x) .
\end{aligned}
$$

This dependence of the local equilibrium parameters on the distribution function is required in order that the BGK equation preserves the conservation laws for number, energy, and momentum.

The boundary conditions are imposed on the half distributions for velocities directed off the walls at $\pm L$ in terms of the corresponding distributions directed into the walls. A formal solution to Eq. (2.1) as a functional of the hydrodynamic variables can be obtained by direct integration, with the integration "constant" (function of $\mathbf{v}$ ) determined by the boundary conditions. The solution for the special case of diffuse boundary conditions has been given in Ref. 8. It is expressed in terms of the dimensionless variables

$$
\begin{gathered}
f^{*}=v_{0}^{5} p_{0}^{-1} f, \quad \mathbf{v}^{*}=\mathbf{v} / v_{0}, \quad s=\left(\lambda / v_{0}\right) \int_{-L}^{x} d x^{\prime} v\left(x^{\prime}\right), \\
\lambda=v_{0}\left[\int_{-L}^{L} d x v(x)\right]^{-1}, \quad T^{*}=T / T_{0}, \quad \mathbf{U}^{*}=\mathbf{U} / v_{0} .
\end{gathered}
$$

Here $v_{0}^{2}=2 T_{0}$ is the thermal velocity at some reference temperature $T_{0}, p_{0}$ is a reference pressure, and $\lambda$ is an average Knudsen number. The domain in terms of the dimensionless space variable $s$ is given by $0<s<1$. In the following it will be understood that dimensionless quantities are considered and the asterisk will be deleted. The solution is then found to be ${ }^{8}$

$$
\begin{aligned}
f(s, \mathbf{v})= & f_{p}(s, \mathbf{v})+f_{b}(s, \mathbf{v}) \\
f_{p}(s, \mathbf{v})= & \int_{0}^{\infty} d t e^{-t} f_{L}\left(s-\lambda v_{x} t, \mathbf{v}\right), \\
f_{b}(s, \mathbf{v})= & \Theta\left(v_{x}\right) n_{-}\left(\pi T_{-}\right)^{-3 / 2} \exp \left[-\left(\mathbf{v}-\mathbf{U}_{-}\right)^{2} / T_{-}\right] e^{-s / \lambda v_{x}}+\Theta\left(-v_{x}\right) n_{+}\left(\pi T_{+}\right)^{-3 / 2} \\
& \quad \times \exp \left[-\left(\mathbf{v}-\mathbf{U}_{+}\right)^{2} / T_{+}\right] e^{-(s-1) / \lambda v_{x}} .
\end{aligned}
$$


The first term on the right-hand side of Eq. (2.7) is a particular solution to Eq. (2.1) corresponding to homogeneous boundary conditions (half distributions vanishing at the boundaries). Thus the specified boundary conditions appear only in the second term, $f_{b}$. The parameters $T_{-}$ and $T_{+}$represent the (constant) temperatures of the walls at $-L$ and $+L$, respectively. Similarly, $U_{ \pm}$ represent the velocities of the walls, taken here as arbitrary constants along the $y$ direction. The constants $n_{ \pm}$ are twice the density of particles coming off the walls and must be determined self-consistently along with the hydrodynamic fields in $f_{p}$.
It is clear that $f_{p}$ has the form of a normal solution, since the space dependence of the $f_{L}$ on the right-hand side of Eq. (2.8) is entirely through the hydrodynamic variables. The additional boundary contribution $f_{b}$ has an explicit space dependence whose range is determined by the average Knudsen number $\lambda$. In the remainder of this section we consider idealized conditions under which this boundary term vanishes, leaving $f_{p}$ as the exact normal solution. One possibility is to choose $T_{ \pm}=0$, $\mathbf{U}_{ \pm}= \pm \hat{\mathbf{y}} U_{0}$. These are conditions for planar Couette flow between very cold walls. It is easily seen that $f_{b}$ vanishes for all $s$ and $v_{x}$ for this choice, leaving

$$
f(s, \mathbf{v})=f_{p}(s, \mathbf{v})=\operatorname{sgn}\left(v_{x}\right)\left(\lambda v_{x}\right)^{-1} \int_{0}^{1} d \sigma \Theta\left((s-\sigma) \operatorname{sgn}\left(v_{x}\right)\right) \exp \left[-(s-\sigma) / \lambda v_{x}\right] f_{L}(\sigma, \mathbf{v}) .
$$

The parameters of the local equilibrium distribution function in the integrand of (2.10) are determined from Eqs. (2.3) - (2.5). Carrying out the integration over $f(s, \mathrm{v})$ using (2.10) gives

$$
\begin{aligned}
& p(s) / T(s)=\lambda^{-1} \int_{0}^{1} d \sigma p(\sigma) T^{-3 / 2}(\sigma) J_{-} 1\left[\frac{\mid s-\sigma}{\lambda \sqrt{T(\sigma)}}\right], \\
& p(s) U_{x}(s) / T(s)=\lambda^{-1} \int_{0}^{1} d \sigma \operatorname{sgn}(s-\sigma) p(\sigma) T^{-1}(\sigma) J_{0}\left(\frac{|s-\sigma|}{\lambda \sqrt{T(\sigma)}}\right), \\
& p(s) U_{y}(s) / T(s)=\lambda^{-1} \int_{0}^{1} d \sigma U_{y}(\sigma) p(\sigma) T^{-3 / 2}(\sigma) J_{-1}\left(\frac{|s-\sigma|}{\lambda \sqrt{T(\sigma)}}\right), \\
& \frac{3}{2} p(s)=\lambda^{-1} \int_{0}^{1} d \sigma p(\sigma) T^{-3 / 2}(\sigma)\left[U_{y}(\sigma)-U_{y}(s)\right]^{2} J_{-1}\left[\frac{|s-\sigma|}{\lambda \sqrt{T(\sigma)}}\right] \\
& \quad+\lambda^{-1} \int_{0}^{1} d \sigma p(\sigma) T^{-1 / 2}(\sigma)\left[J_{-1}\left[\frac{|s-\sigma|}{\lambda \sqrt{T(\sigma)}}\right]+J_{1}\left[\frac{|s-\sigma|}{\lambda \sqrt{T(\sigma)}}\right]\right) .
\end{aligned}
$$

The equation for the $z$ component of the flow velocity has not been given since the choice $U_{z}=0$ is manifestly a solution to (2.5) using (2.10). The functions $J_{m}(x)$ are defined by

$$
J_{m}(x)=(\pi)^{-1 / 2} \int_{0}^{\infty} d t t^{m} e^{-t^{2}} e^{-x / t} .
$$

Some of their properties and values are given in the Appendix of Ref. 8. Equations (2.11)-(2.14) are a set of nonlinear, singular integral equations for the fields $p(s), T(s)$, and $\mathrm{U}(s)$. Once they are determined the distribution function (2.10) is also fully determined.

Uniqueness of solutions to Eqs. (2.11) - (2.14) (if they exist) is determined also by boundary conditions on $p, T$, and $\mathrm{U}$. They can be imposed by requiring that the temperature vanish at the two walls and that the flow field match the velocity of the walls. More generally, it might be assumed that there is some "slip" between the wall conditions and those for $T(s)$ and $\mathbf{U}(s)$. From the symmetry of the problem, the simplest form consistent with these conditions is the following:

$$
p(s)=\text { const, } \quad \mathbf{U}(s)=\hat{\mathbf{y}}\left(-U_{0}+\alpha s\right), \quad T(s)=\beta s(1-s),
$$

where $\alpha=2 U_{0}$ and $\beta$ is a constant to be determined. It is now necessary to verify that these forms satisfy Eqs. (2.11)-(2.14). Consider first Eq. (2.11), which becomes

$$
\lambda / T(s)=\int_{0}^{s} d \sigma T^{-3 / 2}(\sigma) J_{-1}\left(\frac{s-\sigma}{\lambda \sqrt{T(\sigma)}}\right)+\int_{s}^{1} d \sigma T^{-3 / 2}(\sigma) J_{-1}\left(\frac{\sigma-s}{\lambda \sqrt{T(\sigma)}}\right) .
$$

A change of variables $\sigma \rightarrow 1-\sigma$ and noting the symmetry $T(\sigma)=T(1-\sigma)$, shows that the right-hand side of (2.17) can be written

$$
\lambda / T(s)=I(s)+I(1-s), \quad I(s) \equiv \int_{0}^{s} d \sigma T^{-3 / 2}(\sigma) J_{-1}\left(\frac{s-\sigma}{\lambda \sqrt{T(\sigma)}}\right) .
$$

Next, change variables to $u=(1-s) \sigma /(1-\sigma) s$ to get 


$$
I(s)=\left[\beta^{2} T(s)\right]^{-1 / 2} \int_{0}^{1} d u u^{-3 / 2}(1-s+s u) J_{-1}\left(\frac{(1-u)}{\sqrt{u}} \frac{\sqrt{T(s)}}{\lambda \beta}\right) .
$$

Then using again the symmetry of $T(s)$,

$$
I(s)+I(1-s)=\left[\beta^{2} T(s)\right]^{-1 / 2} \int_{0}^{1} d u u^{-3 / 2}(1+u) J_{-1}\left(\frac{(1-u)}{\sqrt{u}} \frac{\sqrt{T(s)}}{\lambda \beta}\right) .
$$

A final change of variables gives the desired result

$$
I(s)+I(1-s)=2 \lambda T^{-1}(s) \int_{0}^{\infty} d t J_{-1}(t)=\lambda / T(s) .
$$

Comparison with (2.18) shows that the assumed forms (2.16) are solutions to Eq. (2.11).

The same changes of variables in Eqs. (2.12) and (2.13) lead to verification that these equations are also solved by the choice (2.16). These solutions apply regardless of the value of the constant $\beta$ in Eq. (2.16) for the temperature $T(s)$. However, a similar analysis of Eq. (2.14) shows that a solution exists only for a particular choice of $\beta$, given implicitly by the equation

$$
\begin{aligned}
& \int_{0}^{\infty} d z\left(1+2 \gamma z^{2}\right)^{-1}\left[J_{1}(z)+\left(1+a^{2} z^{2}\right) J_{-1}(z)\right]=\frac{3}{4} \\
& \gamma(a) \equiv \beta \lambda^{2} / 2, \quad a \equiv \lambda \alpha=\lambda \partial U_{y} / \partial s .
\end{aligned}
$$

The details of the derivation of this result are given in Appendix A. This completes the verification that the assumed forms, (2.16), are indeed solutions to the integral equations (2.11)-(2.14). Accordingly, the distribution function given by (2.10) with the hydrodynamic fields (2.16) is an exact solution to the nonlinear BGK equation for the chosen boundary conditions.

The interpretation of $\gamma(a)$ in (2.23) can be obtained from consideration of the macroscopic conservation laws. As shown in Ref. 6, the hydrodynamic fields (2.16) result from macroscopic heat and momentum fluxes that have the same form as the Navier-Stokes approximation, except with a shear rate dependent thermal conductivity $\kappa(a)$ and a shear viscosity $\eta(a)$ [for consistency with the notation in Ref. 6, the uniformity parameter (shear rate) is identified with $a=\lambda \alpha$ ]. It was shown that $\gamma(a)$ is proportional to the Prandtl number $P(a)$ :

$$
\gamma(a)=a^{2} P(a) / 5=a^{2} \eta(a) / 2 \kappa(a)
$$

It is shown in Appendix A that the definition of $\gamma(a)$ obtained here is the same as that of Ref. 6. Thus the calculation of $\gamma(a)$ given there applies here as well. Also, the above calculations provide a justification of the purely formal manipulation of divergent series in Ref. 6.

The distribution function is completely determined now by substitution of the hydrodynamic fields, (2.16), into the formal solution (2.10). The result depends on $\mathbf{v}$, $s, \lambda$, and $a$. As a normal solution the dependence on $s$ appears only through the hydrodynamic fields. In the present case the hydrodynamic fields are sufficiently simple that the dependence on $s, \lambda$, and $a$ can be expressed entirely in terms of two uniformity parameters $a$ and $\epsilon$. The shear rate $a$, given by Eq. (2.23), is a measure of the spatial variation for the flow field, while $\epsilon$ is a corresponding measure of the temperature variation,

$$
\epsilon=\lambda[T(s)]^{-1 / 2} \partial T(s) / \partial s .
$$

The distribution function then takes the form

$$
\begin{aligned}
& \phi(\xi, \epsilon, a) \equiv f / f_{L} \\
&=\frac{2 \delta(1+\delta)^{3 / 2}}{\epsilon\left|\xi_{x}\right|} e^{\xi^{2}} \int_{0}^{2 /(1-\delta)} d t \Theta\left((1-t) \operatorname{sgn} \xi_{x}\right)\left[2 t-(1-\delta) t^{2}\right]^{-5 / 2} \\
& \times e^{-2 \delta(1-t) / \epsilon \xi_{x}(1+\delta)} \exp \left\{-\frac{1+\delta}{2 t-(1-\delta) t^{2}}\left[\xi_{x}^{2}+\xi_{z}^{2}+\left[\xi_{y}+\frac{2 a \delta}{\epsilon(1+\delta)}(1-t)\right]^{2}\right]\right\}
\end{aligned}
$$

where $\delta(\epsilon, a)=\epsilon /\left(\epsilon^{2}+8 \gamma\right)^{1 / 2}$ and $\xi=[\mathbf{v}-\mathbf{U}(s)] / \sqrt{T(s)}$.

With the distribution function now known, all properties of interest can be calculated by quadratures. Since the fluxes have been discussed in detail elsewhere, ${ }^{6}$ attention here will be restricted to illustrating the form of the distribution function at large shear rates. Before doing so we make some comments regarding the idealized boundary conditions under which it was derived. The initial step was to eliminate the explicit boundary term $f_{b}$ in
Eq. (2.7) by setting the temperature to zero at both walls. This approach is similar to the derivation of an exact solution for steady heat flow ${ }^{7}$ where the boundary terms were eliminated by setting the temperature equal to zero on one boundary and infinite at the other. In that case it was necessary to imagine an unbounded system, whereas here the finite geometry is retained. [The pure heat flow solution is a special case of (2.26) for $a=0$ at constant $\epsilon$; the interpretation of this leads to the unbounded system.] 


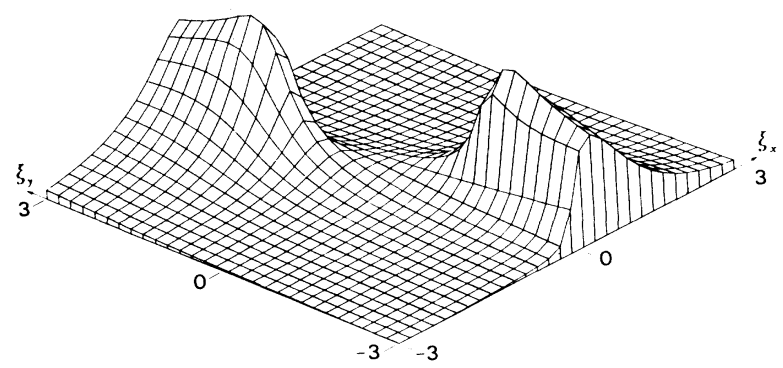

FIG. 1. Reduced distribution function $\psi\left(\xi_{x}, \xi_{y}, \epsilon, a\right)$ for $\epsilon=0$ and $a=1$; maximum value is approximately 6.3 .

As discussed above, and shown by (2.8), the remaining part of the solution, $f_{p}$, depends on the space variable entirely through the hydrodynamic fields. Thus we have constructed explicitly conditions for which the normal solution is exact. These conditions can be viewed as the small Knudsen number limit of a more general class of realistic boundary conditions in the following sense. The relevant local Knudsen number is given by $K(s)=\lambda \sqrt{T(s)}$. Near the walls this measures the size of the boundary layer. By taking the temperature to zero in this region the boundary layer is made asymptotically small.

To illustrate the distortion of the velocity distribution function for states far from equilibrium we consider only the value $a=1$, and calculate the reduced distribution function $\psi\left(\xi_{x}, \xi_{y}, \epsilon, a\right)$ obtained by integrating out the $z$ component of the velocity:

$$
\psi\left(\xi_{x}, \xi_{y}, \epsilon, a\right)=\int d \xi_{z} f / \int d \xi_{z} f_{L} .
$$

Figure 1 shows $\psi$ for the special solution obtained in this section, at $\epsilon=0$. This corresponds to the center of the system where the temperature gradient is locally zero. Figure 2 shows the same distribution for $\epsilon=0.2$, a domain with relatively large temperature gradient. In both cases the system is far from equilibrium and large deviations from equilibrium are observed. The result for $\epsilon=0$ is symmetric with respect to reflection of the velocity. This symmetry is broken by the temperature gradient for $\epsilon>0$, where there are more particles with negative $\xi_{x}$ than with positive $\xi_{x}$. This implies a negative heat flux.

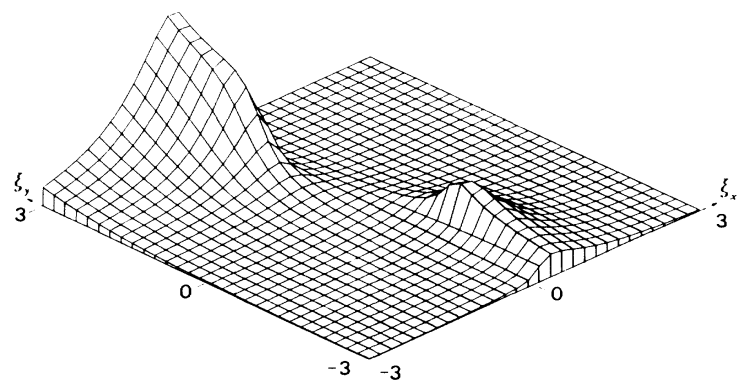

FIG. 2. Same as Fig. 1, for $\epsilon=0.2$; maximum value is approximately 12.9 .
Similarly, for $\epsilon \geq 0$ particles with $\xi_{y}>0$ tend to have $\xi_{x}<0$, and vice versa. This leads to a negative shear stress [Eq. (3.8) below].

\section{FINITE KNUDSEN NUMBER RESULTS}

In this section we consider the more realistic case of walls at finite temperatures. For simplicity, the temperatures at both walls are chosen to be the same, and the local temperature is normalized to the wall temperature (i.e., $T_{+}=T_{-}=T_{0}$ ). The reference pressure is chosen to be $p_{0}=n_{-} T_{0}$. The solution to the BGK equation is given by (2.7), where both $f_{p}$ and the boundary contribution $f_{b}$ are nonzero. The hydrodynamic fields $p(x)$, $T(x)$, and $U(x)$ are again determined from their definitions (2.3) $-(2.5)$. The integration over velocities can be performed, leading to integral equations like (2.11)-(2.14), except that there are now additional contributions from $f_{b}$ on the right-hand sides of these equations. For example, Eq. (2.11) becomes

$$
\begin{aligned}
p(s) / T(s)= & \lambda^{-1} \int_{0}^{1} d \sigma p(\sigma) T^{-3 / 2}(\sigma) J_{-1}\left(\frac{|s-\sigma|}{\lambda \sqrt{T(\sigma)}}\right) \\
& +n_{+} J_{0}((1-s) / \lambda)+J_{0}(s / \lambda) .
\end{aligned}
$$

The last two terms on the right-hand side are responsible for boundary layers near the walls. They decrease away from the wall for $s(1-s) \gg \lambda^{2} \sqrt{T_{+} T_{-}}$, according to the function $J_{0}$. However, the latter does not decay exponentially fast, as the boundary contribution to the distribution function do, so the equations for the hydrodynamic fields provide a more restrictive test for the importance of the boundary layer. This is simply a reflection of the fact that the boundary layer defined by $f_{b}$ depends also on the velocity, and the hydrodynamic boundary layer represents an average over these values. The additional integral equations for $p(s)$ and $\mathbf{U}(s)$ have a structure similar to that of (3.1) and are given in Appendix B. The constant $n_{+}$in (3.1) is determined from these equations as well. They form a closed set of equations whose solutions are obtained numerically using a method described in the Appendix of Ref. 8. Once the hydrodynamic fields are known the distribution function can be calculated using (2.7). Other properties of interest, such as the heat and momentum fluxes, can be calculated from (2.7) as well.

The method for numerical solution of these singular integral equations ${ }^{8}$ is rapidly convergent for $\lambda>1$ over a wide range of shear rates. However, we are mainly interested here in small $\lambda$ to investigate the approach to a normal solution. In this case the method of solution is limited to relatively small shear rates, $a<0.35 \lambda$. For comparison of the results of this section with the special solution of the last section, it is sufficient at these small shear rates to give the results of an asymptotic expansion of (2.26). ${ }^{6}$ This small shear rate expansion at zero Knudsen number yields precisely the formal solution generated by the Chapman-Enskog method. Consequently, this asymptotic form will be referred to in the following as the Chapman-Enskog solution. For comparison with the numerical results of this section, the Chapman-Enskog re- 
sults for the distribution function and two components of the pressure tensor are given [evaluated at the midpoint $s=\frac{1}{2}$, where $\left.\partial T(s) / \partial s=0\right]$ :

$$
\begin{aligned}
& \left(f / f_{L}\right)_{\mathrm{CE}}=1-2 \xi_{x} \xi_{y} a+4 \xi_{x}^{2}\left(\xi_{y}^{2}-\xi^{2} / 5\right) a^{2}+\cdots, \\
& \left(p_{x y} / p\right)_{\mathrm{CE}}=-a+\frac{18}{5} a^{3}+\cdots, \\
& \left(p_{x x} / p\right)_{\mathrm{CE}}=1-\frac{6}{5} a^{2}+\frac{1368}{125} a^{4}+\cdots .
\end{aligned}
$$

The uniformity parameter $a$ is the local shear rate proportional to the velocity gradient [second equality of Eq. (2.23)]. Terms in this expansion up through first order in $a$ are known as Navier-Stokes, while higher-order contributions are referred to as Burnett, super-Burnett, etc. Some comments on the convergence of this expansion are given in the last section.

Returning to the finite Knudsen number problem, we consider first the hydrodynamic fields. It follows directly from the BGK equation that they have the symmetries

$$
\begin{aligned}
& T(1-s)=T(s), \\
& p(1-s)=p(s), \\
& U_{y}(1-s)=-U_{y}(s) .
\end{aligned}
$$

In particular, the special solution of the preceding section has these symmetries. Figure 3 shows the temperature profile at an imposed "shear rate," $2 U_{0}=0.35$ for several values of the average Knudsen number $\lambda$. Figure 4 represents the corresponding results for the velocity field. The pressure is spatially constant to within less than $1 \%$ and is not shown. The qualitative features are the same for all cases, and can be understood in terms of the corresponding Navier-Stokes hydrodynamic approximation. The diffuse boundary conditions used to obtain (2.9) correspond to "stick" boundary conditions for the NavierStokes (NS) equations. The resulting solution is just that given by Eq. (2.16). To compare with the results here at finite Knudsen number, these solutions can be modified by an effective "slip" at the boundary obtained by adding

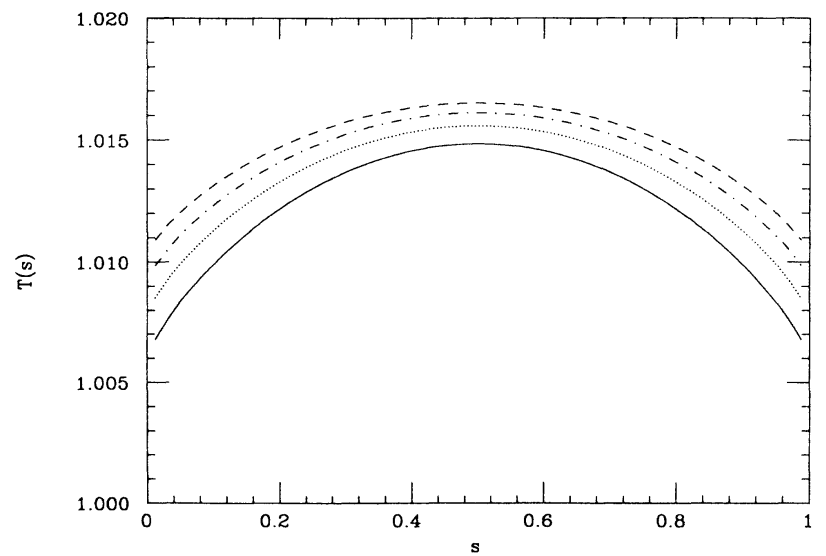

FIG. 3. Temperature, $T(s)$, for $\lambda=0.2(-), \lambda=0.3(\cdots)$, $\lambda=0.4(-\cdot-\cdot)$, and $\lambda=0.5(---)$ at $2 U_{0}=0.35$.

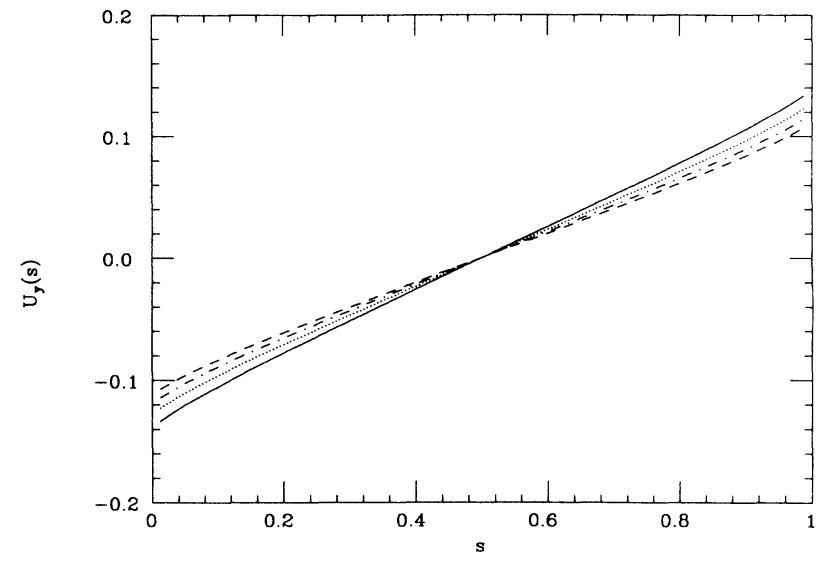

FIG. 4. Flow velocity profiles for the same parameters as Fig. 3.

a constant velocity $\Delta U$ to the flow field $U_{y}$ and a constant temperature $\Delta T$ to the temperature. The solutions then become

$$
\begin{aligned}
& p_{\mathrm{NS}}(s)=\text { const }, \\
& T_{\mathrm{NS}}(s)=1+\Delta T+\frac{2}{5} \alpha^{2} s(1-s), \\
& \mathbf{U}_{\mathrm{NS}}(s)=\mathbf{y}\left(-U_{0}+\Delta U+\alpha s\right),
\end{aligned}
$$

where the effective slope of the velocity curves, $\alpha$, is determined from Fig. 4 at $s=0.5$. With these modifications of the Navier-Stokes solutions there is very good agreement with Figs. 3 and 4. In particular, the velocity field is very nearly linear for all $\lambda$, and the temperature profiles deviate from (3.6) by less than $0.02 \%$ for all $\lambda \leq 0.5$. This means that the primary influence of finite Knudsen number on the hydrodynamic profiles is simply a velocity and temperature slip at the boundary. The values of the slip can be compared with theoretical calcu-

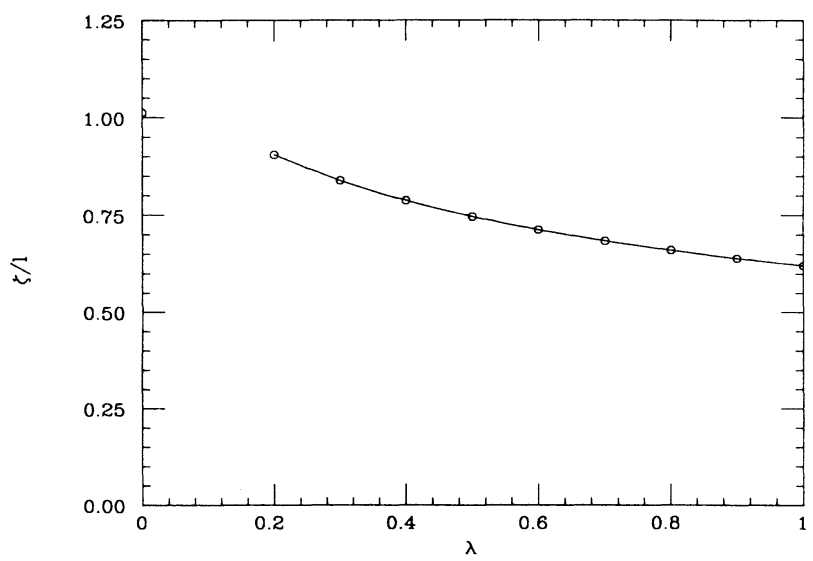

FIG. 5. Slip coefficient as a function of Knudsen number for $2 U_{0}=0.35$; the point at $\lambda=0$ indicates the theoretical value at zero Knudsen number. 
lations at small Knudsen number and shear rate. ${ }^{13}$ For example, the velocity slip is usually characterized by a slip length $\zeta$ :

$$
\Delta U=\zeta \frac{\partial U_{N S}}{\partial x} \text { or } \zeta / l=\Delta U / \lambda \alpha
$$

where $l=v_{0} / v(x)$ is the mean free path. Figure 5 shows the calculated slip coefficient $\xi / l$ as a function of $\lambda$ for $2 U_{0}=0.35$. These results extrapolate reasonably well to the $\lambda=0$ theoretical value of 1.012 .

Next we consider the fluxes. The exact expressions for the fluxes are obtained from calculating appropriate moments of the distribution function (2.7). For example, the $x y$ component of the pressure tensor is found to be

$$
\begin{aligned}
p_{x y}(s)= & \int d \mathbf{v} v_{x}\left(v_{y}-U_{y}\right) f(s, \mathbf{v}) \\
= & -2 \lambda^{-1} \int_{0}^{1} d \sigma p(\sigma) T^{-1}(\sigma)\left|U_{y}(s)-U_{y}(\sigma)\right| J_{0}\left(\frac{|s-\sigma|}{\lambda \sqrt{T(\sigma)}}\right)-2 n_{+} J_{1}((1-s) / \lambda)\left[U_{0}-U_{y}(s)\right] \\
& -2 J_{1}(s / \lambda)\left[U_{0}+U_{y}(s)\right] .
\end{aligned}
$$

Figure 6 shows a comparison of the results obtained from (3.8) with the Chapman-Enskog expansion, (3.3), at Navier-Stokes order. The results are shown as a function of $\lambda$ for $2 U_{0}=0.1$. The agreement for $\lambda \leq 0.2$ is excellent, while for larger $\lambda$ boundary effects lead to discrepancies.

These results indicate that a normal solution might be expected for $\lambda \leq 0.2$. It would be interesting to know if this data supports the Chapman-Enskog solution beyond the Navioer-Stokes approximation. Figure 7 shows the same results for the shear stress component as a function of $a$ at $\lambda=0.2$. For the smaller values of the shear rate the curve is very nearly linear with a slope equal to the Navier-Stokes value of -1 . At the higher values of $a$ there is a deviation from linearity with the same sign as that of (3.3), but there is not sufficient accuracy to isolate the Chapman-Enskog coefficient of order $a^{3}$ from boundary effects. The corrections to Navier-Stokes for the normal stress $p_{x x}$ occur at order $a^{2}$ and the associated bulk coefficient can be determined with greater accuracy. The exact expression for $p_{x x}$ is

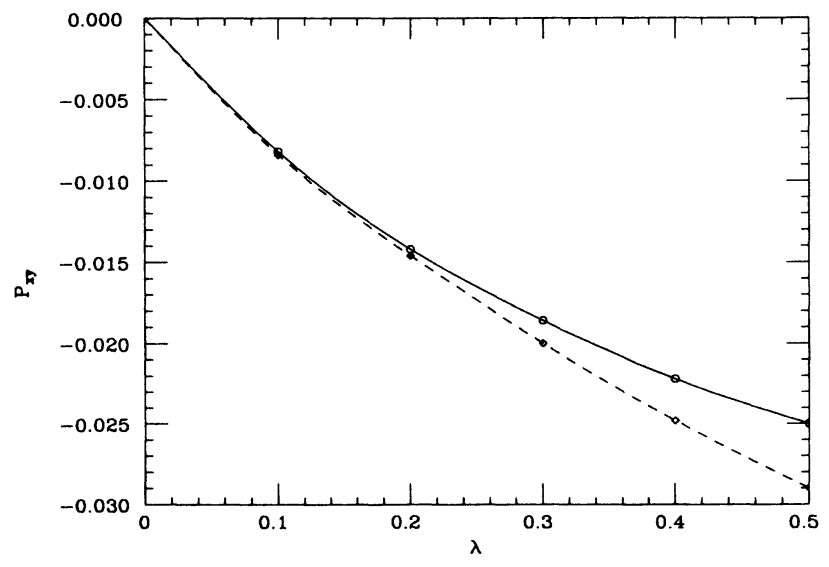

FIG. 6. Pressure tensor ( $x y$ component) as a function of Knudsen number for $2 U_{0}=0.1$; numerical $(-)$ and Newton's viscosity law $(---)$.

$$
\begin{aligned}
p_{x x}(s)= & \int d \mathbf{v} v_{x}^{2} f(s, \mathbf{v}) \\
= & 2 \lambda^{-1} \int_{0}^{1} d \sigma p(\sigma) T^{-1 / 2}(\sigma) J_{1}\left(\frac{|s-\sigma|}{\lambda \sqrt{T(\sigma)}}\right) \\
& +2 n_{+} J_{2}((1-s) / \lambda)+2 J_{2}(s / \lambda) .
\end{aligned}
$$

The results from numerical evaluation of (3.9) are shown in Fig. 8 for $\lambda=0.2$. A clear linear dependence on $a^{2}$ is obtained, but the slope is approximately 0.94 instead of the Chapman-Enskog value of $6 / 5$ in Eq. (3.4). This discrepancy can be understood as a Knudsen number dependence, as shown in Fig. 9. Extraplation to zero Knudsen number shows agreement within the accuracy of the calculation. Thus we believe these results support the validity of the Chapman-Enskog normal solution beyond Navier-Stokes order.

Finally, we consider the distribution function $f(s, \mathbf{v})$. The reduced distribution function defined in Eq. (2.27) is shown in Fig. 10. As expected, there is good agreement with the Chapman-Enskog solution (3.2) to Navier-

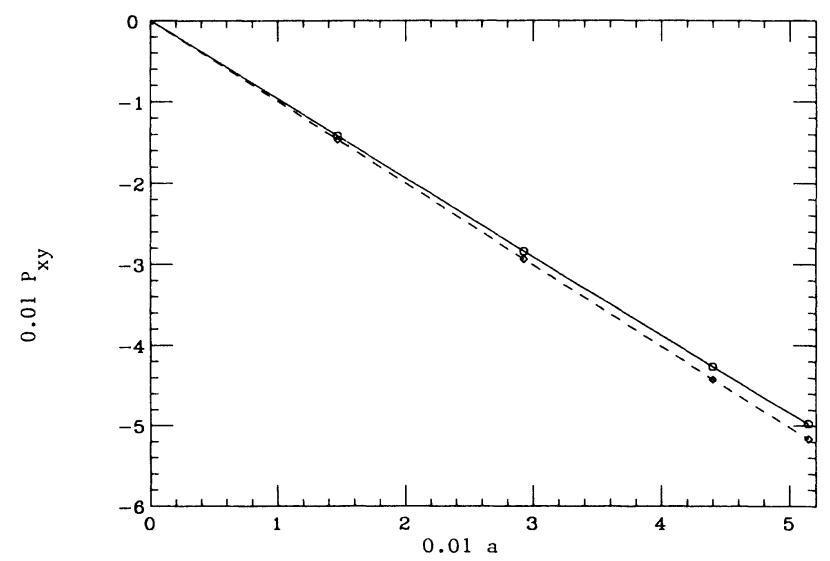

FIG. 7. Same as Fig. 6 as a function of shear rate for $\lambda=0.2$. 


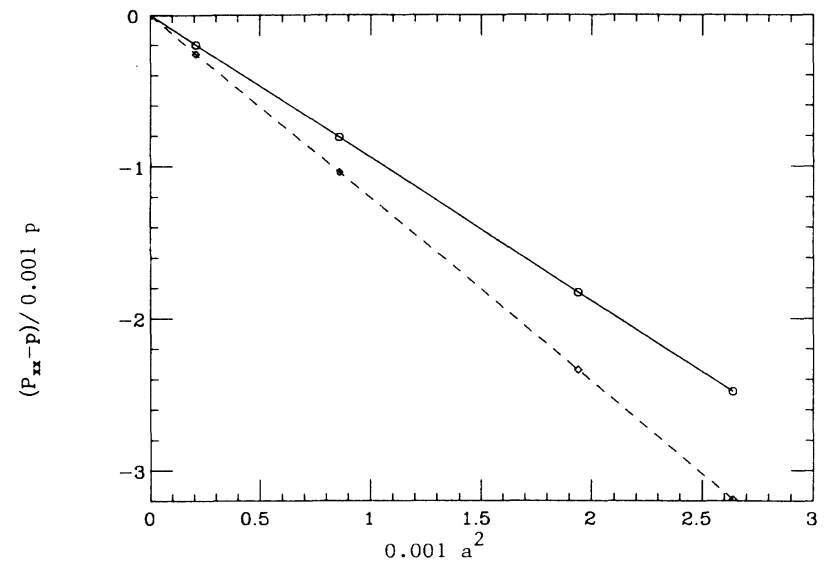

FIG. 8. Normal stress ( $x x$ component) as a function of $a^{2}$ for $\lambda=0.2$; numerical ( - ) and Burnett order ( ---$)$.

Stokes order for $\left|\xi_{x}\right| \leq 1$. At larger velocities the effective boundary layer extends into the bulk of the system and finite Knudsen number deviations are observed.

\section{DISCUSSION}

The analysis of Couette flow described in the previous two sections is essentially exact. In Sec. II an analytic solution is obtained for boundary conditions corresponding to zero Knudsen number, but arbitrary shear rate. This constitutes an explicit construction of a normal solution without limitation to states near equilibrium. The asymptotic expansion of this solution agrees with the Chapman-Enskog construction for small shear rates. Since the latter is expected to be divergent but asymptotic, ${ }^{7,10,14}$ the analysis here associates that asymptotic expansion with a well-defined solution to the kinetic equation. Similar results were obtained for pure heat flow.

A formally exact solution for diffuse boundary condi-

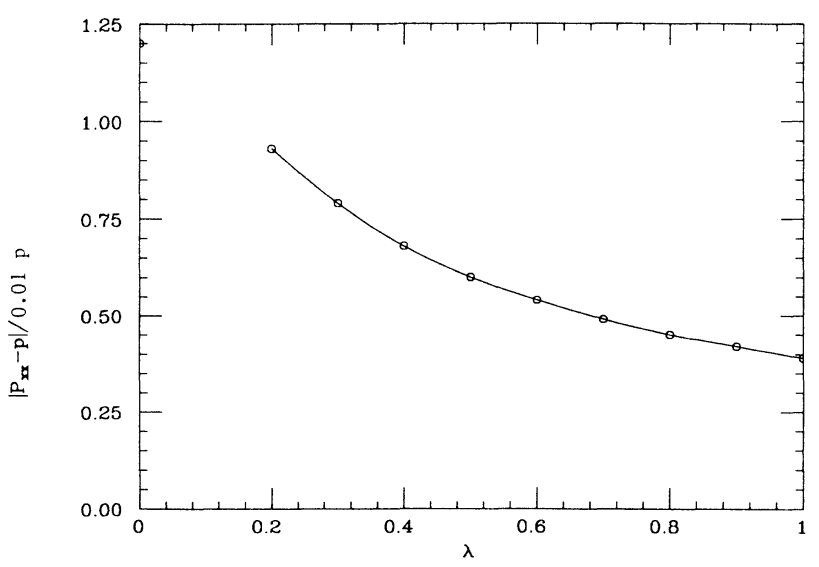

FIG. 9. Same as Fig. 8, except as a function of Knudsen number for $2 U_{0}=0.35$.

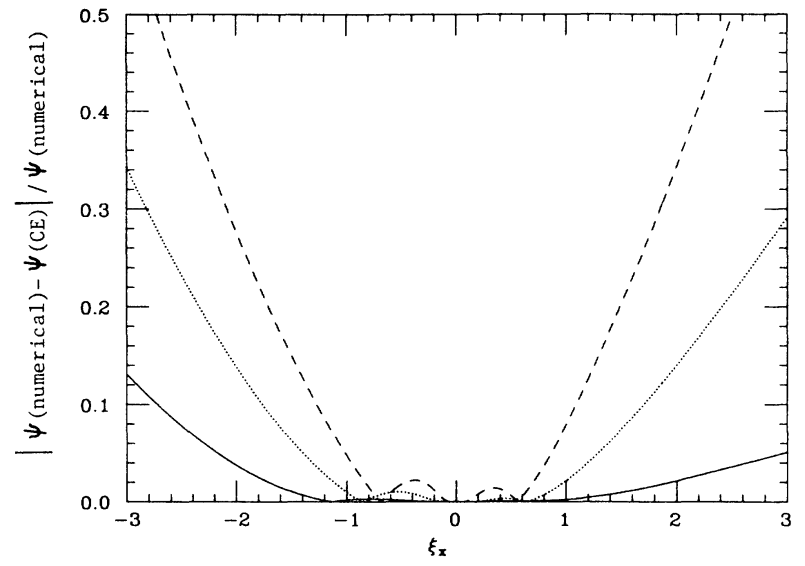

FIG. 10. Relative ratio of reduced distribution functions: $\mid \psi($ numerical $)-\psi(\mathrm{CE}) / \psi($ numerical $) \mid$ for $2 U_{0}=0.35, s=0.5$, and $\xi_{y}=0.83 ; \lambda=0.2(-), \lambda=0.5(\cdots)$, and $\lambda=1.0(---)$.

tions, (2.7), was evaluated by numerical solution to the integral equations for the temperature, pressure, and flow velocity in Sec. III. The diffuse boundary conditions lead to boundary layers, reflected in a Knudsen number dependence of the hydrodynamic fields, fluxes, and distribution function. The numerical method used is not applicable at very small Knudsen numbers for large shear rates, but the approach to a normal solution was studied by considering properties as a function of Knudsen number. Specifically, it was found that the shear stress component $p_{x y}$ is well described by the Navier-Stokes approximation for $\lambda \leq 0.2$. Furthermore, the normal pressure component $p_{x x}$ was found to agree with the ChapmanEnskog result up through Burnett order. Numerical accuracy of the method for solving the integral equations was monitored by checking that $p_{x y}$ is spatially constant (an exact property from the BGK equation) to within $1 \%$.

The hydrodynamic fields were found to agree very well with the zero Knudsen number form, if appropriate slip coefficients were used to modify the boundary conditions. The slip coefficient for the velocity also was found to agree well with that calculated from theory.

The distribution function for zero Knudsen number was calculated for large shear rates to illustrate its distortion far from equilibrium. It was also calculated for finite Knudsen number and small shear rates for comparison with the Chapman-Enskog result. As expected, agreement in the latter case was observed for velocities less than the local thermal velocity.

\section{ACKNOWLEDGMENTS}

Support from the Division of Sponsored Research at the University of Florida is gratefully acknowledged by C.S.K. Two of us (A.S. and J.J.B.) acknowledge partial support from the Direccion General de Investigacion Científica y Técnica (Spain) through Grant No. PB 860205. 
APPENDIX A: PROOF OF EQ. (2.22)

Equation (2.14) imposes a relationship between the parameters $\alpha$ and $\beta$ in the assumed forms for the velocity and temperature fields of (2.16). The resulting relationship, given by Eq. (2.22), is verified in this appendix. Equation (2.14) is

$$
\begin{aligned}
\frac{3}{2} p(s)= & \lambda^{-1} \int_{0}^{1} d \sigma p(\sigma) T^{-3 / 2}(\sigma)\left[U_{y}(\sigma)-U_{y}(s)\right]^{2} J_{-1}\left(\frac{|s-\sigma|}{\lambda \sqrt{T(\sigma)}}\right) \\
& +\lambda^{-1} \int_{0}^{1} d \sigma p(\sigma) T^{-1 / 2}(\sigma)\left[J_{-1}\left(\frac{|s-\sigma|}{\lambda \sqrt{T(\sigma)}}\right)+J_{1}\left(\frac{|s-\sigma|}{\lambda \sqrt{T(\sigma)}}\right)\right] .
\end{aligned}
$$

Using the explicit forms for $T, p$, and $\mathbf{U}$ given in (2.16) this can be written

$$
\begin{aligned}
& \frac{3}{2}=i(s)+i(1-s), \\
& i(s)=\lambda^{-1} \int_{0}^{s} d \sigma T^{-1 / 2}(\sigma)\left[\left(1+\frac{\alpha^{2}(\sigma-s)^{2}}{T(\sigma)}\right) J_{-1}\left(\frac{|s-\sigma|}{\lambda \sqrt{T(\sigma)}}\right)+J_{1}\left(\frac{|s-\sigma|}{\lambda \sqrt{T(\sigma)}}\right)\right] .
\end{aligned}
$$

Next a change of variables to $u=(1-s) \sigma /(1-\sigma) s$ gives

$$
\begin{aligned}
& i(s)=T^{1 / 2}(s)(\lambda \beta)^{-1} \int_{0}^{1} d u u^{-1 / 2}(1-s+s u)^{-1}\left[J_{1}\left[\frac{(1-u)}{\sqrt{u}} \frac{\sqrt{T(s)}}{\lambda \beta}\right]+\left(1+\frac{T(s) \alpha^{2}(1-u)^{2}}{\beta^{2} u}\right] J_{-1}\left[\frac{(1-u)}{\sqrt{u}} \frac{\sqrt{T(s)}}{\lambda \beta}\right]\right) \text {, } \\
& i(s)+i(1-s)=T^{1 / 2}(s)(\lambda \beta)^{-1} \int_{0}^{1} \frac{d u}{\sqrt{u}} \frac{u+1}{s(1-s)\left(1+u^{2}\right)+u\left(1-2 s+2 s^{2}\right)} \\
& \times\left[J_{1}\left(\frac{(1-u)}{\sqrt{u}} \frac{\sqrt{T(s)}}{\lambda \beta}\right]+\left[1+\frac{T(s) \alpha^{2}(1-u)^{2}}{\beta^{2} u}\right] J_{-1}\left[\frac{(1-u)}{\sqrt{u}} \frac{\sqrt{T(s)}}{\lambda \beta}\right]\right] .
\end{aligned}
$$

Finally, a change of variables to $z=T^{1 / 2}(\lambda \beta)^{-1}(1$ $-u) u^{-1 / 2}$ leads to

$$
\begin{aligned}
i(s)+i & (1-s) \\
& =2 \int_{0}^{\infty} d z\left(1+\gamma z^{2}\right)^{-1}\left\{J_{1}(z)+\left[1+(a z)^{2}\right] J_{-1}(z)\right\},
\end{aligned}
$$

where $a=\alpha \lambda$ and $\gamma=\lambda^{2} \beta / 2$. Combining (A6) with (A2) gives Eq. (2.22):

$$
\frac{3}{4}=\int_{0}^{\infty} d z\left(1+2 \gamma z^{2}\right)^{-1}\left\{J_{1}(z)+\left[1+(a z)^{2}\right] J_{-1}(z)\right\} .
$$

Next we verify that this implicit definition of $\gamma(a)$ is the same as that found in Ref. 6. First (A7) is rewritten as

$$
\begin{aligned}
& a^{2}-3 \gamma-\left(a^{2}-3 \gamma\right) F_{1}(\gamma)+2 \gamma^{2} F_{2}(\gamma)=0 \\
& F_{1}(\gamma) \equiv 2 \int_{0}^{\infty} d z\left(1+2 \gamma z^{2}\right)^{-1} J_{-1}(z) \\
& F_{2}(\gamma) \equiv \gamma^{-1} \int_{0}^{\infty} d z\left(1+2 \gamma z^{2}\right)^{-1}\left[2 J_{1}(z)-J_{-1}(z)\right]
\end{aligned}
$$

(A10)
The function $F_{1}(\gamma)$ can be written more explicitly using (2.15):

$$
\begin{aligned}
F_{1}(\gamma) & =\frac{2}{\sqrt{\pi}} \int_{0}^{\infty} d z e^{-z} \int_{0}^{\infty} d t\left(1+2 \gamma z^{2}\right)^{-1} e^{-t^{2}} \\
& =\int_{0}^{\infty} d z e^{-z} \int_{0}^{\infty} d t e^{-t} e^{-(z t)^{2} \gamma / 2}
\end{aligned}
$$

where use has been made of identity 7.4.11 in Ref. 15 . Then, by a similar analysis and the identity $\partial J_{1}(z) / \partial z=-J_{0}(z)$ it is possible to show that $F_{2}(\gamma)$ is the derivative of $F_{1}(\gamma)$. With these results, Eq. (A8) is seen to be the same as Eqs. (3.14) and (3.15) of Ref. 6.

\section{APPENDIX B: INTEGRAL EQUATIONS FOR DIFFUSE BOUNDARY CONDITIONS}

In this appendix we given the complete set of integral equations for the temperature, pressure, and flow velocity in the case of diffuse boundary conditions:

$$
p(s) / T(s)=\lambda^{-1} \int_{0}^{1} d \sigma p(\sigma) T^{-3 / 2}(\sigma) J_{-1}\left[\frac{|s-\sigma|}{\lambda \sqrt{T(\sigma)}}\right]+n_{+} J_{0}\left((1-s) / \lambda \sqrt{T_{+}}\right)+n_{-} J_{0}\left(s / \lambda \sqrt{T_{-}}\right)
$$




$$
\begin{aligned}
& p(s) U_{x}(s) / T(s)=0=\lambda^{-1} \int_{0}^{1} d \sigma p(\sigma) T^{-1}(\sigma) \operatorname{sgn}(s-\sigma) J_{0}\left[\frac{|s-\sigma|}{\lambda \sqrt{T(\sigma)}}\right]-n_{+} \sqrt{T_{+}} J_{1}\left((1-s) / \lambda \sqrt{T_{+}}\right) \\
& +n_{-} \sqrt{T_{-}} J_{1}\left(s / \lambda \sqrt{T_{-}}\right) \\
& p(s) U_{y}(s) / T(s)=\lambda^{-1} \int_{0}^{1} d \sigma p(\sigma) T^{-3 / 2}(\sigma) U_{y}(\sigma) J_{-1}\left[\frac{|s-\sigma|}{\lambda \sqrt{T(\sigma)}}\right]+n_{+} U_{+} J_{0}\left((1-s) / \lambda \sqrt{T_{+}}\right) \\
& -n_{-} U_{-} J_{0}\left(s / \lambda \sqrt{T_{-}}\right) \\
& \frac{3}{2} p(s)=\lambda^{-1} \int_{0}^{1} d \sigma p(\sigma) T^{-3 / 2}(\sigma)\left[U_{y}(\sigma)-U_{y}(s)\right]^{2} J_{-1}\left(\frac{|s-\sigma|}{\lambda \sqrt{T(\sigma)}}\right) \\
& +\lambda^{-1} \int_{0}^{1} d \sigma p(\sigma) T^{-1 / 2}(\sigma)\left[J_{-1}\left(\frac{|s-\sigma|}{\lambda \sqrt{T(\sigma)}}\right)+J_{1}\left(\frac{|s-\sigma|}{\lambda \sqrt{T(\sigma)}}\right]\right] \\
& +n_{+}\left[\left(T_{+}+U_{+}^{2}\right) J_{0}\left((1-s) / \lambda \sqrt{T_{+}}\right)+T_{+} J_{2}\left((1-s) / \lambda \sqrt{T_{+}}\right)\right] \\
& +n_{-}\left[\left(T_{-}+U_{-}^{2}\right) J_{0}\left(s / \lambda \sqrt{T_{-}}\right)+T_{-} J_{2}\left(s / \lambda \sqrt{T_{-}}\right)\right] .
\end{aligned}
$$

These equations are solved numerically using the method described in the Appendix of Ref. 8 .

1J. R. Dorfman and H. van Beijeren in Statistical Mechanics, Part B: Time Dependent Processes, edited by B. Bern (Plenum, New York, 1977).

${ }^{2}$ C. Trozzi and G. Ciccotti, Phys. Rev. A 29, 916 (1984); A. Tennenbaum, G. Ciccotti, and R. Gallico, ibid. 25, 2778 (1982).

${ }^{3}$ P.-J. Clause and M. Mareschal, Phys. Rev. A 38, 4241 (1988); D. K. Bhattacharya and G. C. Lie, Phys. Rev. Lett. 62, 897 (1989).

${ }^{4}$ J. Gómez Ordóñez, J. J. Brey, and A. Santos, Phys. Rev. A 39, 3038 (1989).

${ }^{5}$ A. Santos, J. J. Brey, and V. Garzo, Phys. Rev. A 34, 5047 (1986).

6J. J. Brey, A. Santos, and J. W. Dufty, Phys. Rev. A 36, 2842 (1987).

${ }^{7}$ A. Santos, J. J. Brey, C. S. Kim, and J. W. Dufty, Phys. Rev. A
39, 320 (1989).

${ }^{8}$ C. S. Kim, J. W. Dufty, A. Santos, and J. J. Brey, Phys. Rev. A 39, 328 (1989).

${ }^{9}$ P. Bhatnagar, E. Gross, and M. Krook, Phys. Rev. 94, 511 (1954).

${ }^{10}$ H. Grad, Phys. Fluids 6, 147 (1963).

${ }^{11} \mathrm{C}$. Cercignani, Theory and Application of the Boltzmann Equation (Elsevier, New York, 1975).

${ }^{12}$ D. R. Willis, Phys. Fluids 5, 127 (1962).

${ }^{13}$ S. Albertoni, c. Cercignani, and L. Gotusso, Phys. Fluids 6, 993 (1963).

${ }^{14}$ A. Santos, J. J. Bery, and J. W. Dufty, Phys. Rev. Lett. 56, 1571 (1986).

${ }^{15}$ M. Abramowitz and I. Stegun, Handbook of Mathematical Tables (Dover, New York, 1972). 